\title{
PERCURSOS EM ZIGUEZAGUE EM ROTAS DE FUGA E A SUA INFLUÊNCIA NO FLUXO DE EVACUAÇÃO DE PEDESTRES
}

\author{
Manuela Marques Lalane Nappi \\ Universidade Federal de Santa Catarina \\ Campus Reitor João David Ferreira Lima, s/n - Trindade, Florianópolis - SC, 88040-900 \\ lalppi.ms@gmail.com \\ João Carlos Souza \\ Universidade Federal de Santa Catarina \\ Campus Reitor João David Ferreira Lima, s/n - Trindade, Florianópolis - SC, 88040-900 \\ joao.carlos@ufsc.br
}

\begin{abstract}
RESUMO
Pesquisas sobre a dinâmica de pedestres são capazes de prever que pequenas características arquitetônicas do entorno podem ter grandes efeitos no comportamento de multidões bem como no fluxo de pedestres. O formato de funil próximo a uma saída, por exemplo, é capaz de evitar um entupimento e percursos em ziguezague são úteis em situações de pânico, ainda que acarretem desconforto, devendo-se analisar o seu custo/benefício para situações normais. A partir de simulações computacionais baseadas no Modelo de Força Social, são analisados neste trabalho três projetos de um ambiente fechado, destinado à reunião de público com concentração. Diferentes desenhos de rotas de fuga buscam comparar a eficácia de percursos em ziguezague em relação a percursos retos, tanto para situações normais quanto de emergência. As simulações realizadas evidenciaram que a presença de percursos em ziguezague após o gargalo não foi capaz de gerar fluxos de evacuação mais eficazes. Já a presença do painel em ziguezague antes do gargalo mostrou-se eficaz nas situações de maior densidade de pessoas, com redução de até $11,23 \%$ no tempo total de evacuação.
\end{abstract}

Palavra-chave: Arquitetura; Design de rotas de fuga; Percursos em ziguezague; Simulação Computacional; Modelo de Força Social.

\begin{abstract}
Research on pedestrian dynamics predict that small architectural features of the surrounding environment can have major effects on crowd behavior as well as pedestrian flow. For example, a funnel shape near an exit, is able to prevent clogging, and zigzag pathways are useful in panic situations, even if they cause discomfort and should be analyzed for cost-effectiveness in normal situations. From computational simulations based on the Social Force Model, this work analyzes three projects of a closed environment, intended for public gatherings. Different designs of escape routes were proposed in order to compare the effectiveness of zigzag pathways and straight routes for both normal and emergency situations. The simulations showed that the presence of zigzag pathways after the bottleneck was not able to generate more effective evacuation flows. In contrast, the presence of the
\end{abstract}


zigzag panel before the bottleneck proved to be effective in situations of higher density of people, with a reduction of up to $11.23 \%$ in the total evacuation time.

Keywords: Architecture; Escape route design; Zigzag pathways; Computational simulation; Social Force Model.

\section{Como Citar:}

NAPPI, Manuela Marques Lalane; SOUZA, João Carlos. Percursos em Ziguezague em Rotas de Fuga e a sua Influência no Fluxo de Evacuação de Pedestres. In: SIMPÓSIO DE PESQUISA OPERACIONAL E LOGÍSTICA DA MARINHA, 19., 2019, Rio de Janeiro, RJ. Anais [...]. Rio de Janeiro: Centro de Análises de Sistemas Navais, 2019.

\section{INTRODUÇÃO}

A dinâmica de pedestres pode ser dividida em situações normais e situações de pânico. Embora os problemas característicos de cada uma dessas situações sejam caracterizados por diferentes fenômenos e investigados por diferentes comunidades científicas, podem ser tratados de maneira consistente por um mesmo modelo [1].

É natural supor que interações inteligentes sejam resultado de um processo de aprendizado que otimizou uma resposta automática, que minimizaria colisões e atrasos [2]. Ocorre que a pesquisa sobre comportamento de pedestres e multidões é altamente multidisciplinar. Ela envolve atividades de cientistas, psicólogos, sociólogos, biólogos, físicos, cientistas da computação, etc. O que explica a existência de opiniões controversas sobre o assunto, como ocorre com o conceito de pânico, com a explicação de padrões de movimento coletivos ou com o melhor conceito de modelagem [2].

A debandada de pedestres representa um comportamento desastroso e geralmente é desencadeada em situações onde se estabelece o risco de morte, como em situações de incêndio em corredores lotados, ou quando há pressa para se chegar a eventos de grande escala. Grandes confraternizações de pedestres são muito frequentes em encontros religiosos de grandes festivais hindus, na Índia ou em Meca, por exemplo [3]. Nestas confraternizações, corriqueiramente presencia-se debandadas de multidões que podem ser atribuídas a numerosas razões. Daí o crescente impulso nas últimas décadas para modelar o comportamento de pedestres $[4,5]$.

Com a capacidade de modelar diversos comportamentos coletivos e possibilitar que um mesmo modelo de pedestres possa tratar de situações normais e de fuga, o modelo de força social é um tipo de abordagem amplamente utilizado [4, 6]. Uma vez calibradas com dados empíricos de fluxos de pedestres, as simulações computacionais baseadas nesse modelo são capazes de produzir resultados realistas, mesmo quando se consideram novas geometrias e situações [7]. Assim, o Modelo de Força Social possui valor preditivo, permitindo a investigação de novos cenários, para os quais os experimentos se tornariam caros, difíceis de serem realizados ou perigosos [7]. Esse valor preditivo torna-se relevante no planejamento e otimização de rotas de fuga.

Pesquisas sobre a dinâmica de pedestres são capazes de prever que pequenas características arquitetônicas do entorno podem ter grandes efeitos no comportamento de multidões bem como no fluxo de pedestres. Esses efeitos são especialmente visíveis quando se trata de multidões em pânico [8]. O formato de funil próximo a uma saída, por exemplo, é capaz de evitar um entupimento e percursos em ziguezague são úteis em situações de pânico, ainda que acarretem desconforto, devendo-se analisar o seu custo/benefício para situações normais [3]. Os resultados alcançados por uma pesquisa alemã confirmam que a eficiência de 
um projeto é um problema multi-objetivo [3]. E os objetivos podem ser muitos: maximização de fluxos, minimização de desconforto, redução do número de feridos, etc.

A partir de simulações computacionais baseadas no Modelo de Força Social, são analisados neste trabalho três projetos de um ambiente fechado, destinado à reunião de público com concentração. Diferentes desenhos de rotas de fuga buscam comparar a eficácia de percursos em ziguezague em relação a percursos retos, tanto para situações normais quanto de emergência. Vale destacar que as simulações foram realizadas para duas diferentes densidades de pedestres, 1 e 2 pessoas por $\mathrm{m}^{2}$, fator que influencia a pressão exercida pela multidão, objeto de preocupação de pesquisas na área. Os resultados encontrados demostram que uma rota de fuga é mais eficaz quando seu desenho se dá em linha reta, se comparado ao desenho em ziguezague. No entanto, um pequeno trecho em ziguezague à montante das saídas atuou de maneira positiva nos cenários cuja densidade era de 2 pessoas $/ \mathrm{m}^{2}$.

\section{MODELO DE FORÇA SOCIAL}

Corriqueiramente o comportamento humano dá sinais de ser caótico, irregular e imprevisível. A razão e as condições para esse comportamento ser modelado por meio de forças fazem com que se considere um sistema onde as oscilações devido a influências desconhecidas não sejam grandes [1]. Segundo os autores, este é o caso do tráfego de pedestres e veículos, onde as pessoas, quando confrontadas com situações padrão, reagem automaticamente e não precisam tomar decisões complicadas entre as várias alternativas possíveis. Cita-se como exemplo o caso de um motorista experiente que não precisa pensar detalhadamente em ações a serem tomadas ao acelerar ou mudar de faixa. Trata-se de um comportamento automático que pode ser interpretado como resultado de um processo de aprendizado [1].

Apesar da aparência caótica do comportamento individual de pedestres, é possível verificar-se regularidades [1]. Com base em suas observações e em outros estudos, os autores resumem-nas da seguinte forma:

a) os pedestres experimentam forte aversão a desvios ou movimentos opostos à direção de caminhada desejada, ainda que o caminho desejado esteja congestionado. Há evidências, no entanto, de que os pedestres normalmente escolham a rota mais rápida para atingir o seu destino e não a mais curta [9]. De maneira geral, os pedestres consideram os desvios e o conforto da caminhada pretendida e, assim, minimizam o esforço para chegar ao seu destino [10].

b) pedestres preferem caminhar com uma velocidade individual desejada, que corresponde àquela mais confortável, desde que seja possível chegar ao seu destino a tempo. As velocidades desejadas dentro de multidões de pedestres são gaussianas, distribuídas com um valor médio de, aproximadamente, $1,34 \mathrm{~m} / \mathrm{s}$ com desvio padrão, também aproximado, de $0,26 \mathrm{~m} / \mathrm{s}$ [11]. A velocidade média depende da situação [12], sexo e idade, da hora do dia, do objetivo da viagem, do entorno, etc. [13].

c) os pedestres tendem a manter determinada distância em relação a outros pedestres e fronteiras, a exemplos de ruas, paredes e obstáculos. Essa distância diminui conforme a pressa e conforme a densidade de pedestres cresce.

Em geral, indivíduos em repouso distribuem-se uniformemente sobre a área disponível, desde que não haja pessoas conhecidas entre eles. A densidade de pedestres só aumenta (distâncias interpessoais diminuem) em torno de lugares atraentes e diminui com a variação crescente de velocidade. Além disso, indivíduos que se conhecem podem formar grupos que passam a se comportar como pedestres isolados. Já em situações de pânico, a debandada é um dos mais trágicos comportamentos coletivos, o que se deve ao fato desse 
comportamento já haver provocado a morte de muitas pessoas por esmagamento ou pisoteamento [1].

O Modelo de Força Social proposto em Helbing et al. [4, 6, 7] e Helbing e Molnar [4] está fundamentado no método de modelagem de multidões fluidas de Henderson [15], conforme afirmam os próprios autores. Trata-se de um modelo contínuo, microscópico, cujas interações determinísticas se baseiam na força. O conceito do modelo está fundamentado no pressuposto de que as mudanças no movimento de pedestres são guiadas por campos de força social. Resumidamente, ele se baseia na sobreposição de efeitos de atração e de repulsão, que são responsáveis por determinar o comportamento dos indivíduos.

O conceito de força social é capaz de reproduzir a maior parte das observações empíricas de maneira simples e natural [2]. Para obter-se uma simulação confiável não é necessário saber para que lado um determinado pedestre irá virar numa interseção, por exemplo. Para isso, seria suficiente contar com uma boa estimativa da porcentagem de pedestres que vira para a direita ou para a esquerda [2]. De certa forma, a incerteza relacionada ao comportamento individual é calculada no nível macroscópico, assim como na dinâmica dos fluidos. No entanto, ao invés de um modelo fluido-dinâmico, o modelo de força social se utiliza de uma abordagem de simulação mais flexível, que se baseia no conceito de força generalizada [1].

O atual campo de simulação de pedestres aponta para três grandes áreas de aplicação. A primeira delas trata da necessidade de estimativas corretas das escolhas de destino e rota e da correta precisão de movimento de multidões [16]. Os autores detectaram diversos modelos com capacidade de simular uma opção de rota global e várias classes de pedestres, especialmente os modelos de rede. No entanto, se fazem necessários desenvolvimentos adicionais para que sejam incorporados todos os fenômenos de movimento de multidão.

Uma segunda área de aplicação se refere à pesquisa desse movimento. Nesse sentido, seis modelos foram capazes de modelar todos os casos bases de movimento, mas nenhum deles conseguiu simular todos os fenômenos de auto-organização. Os modelos descritos nos trabalhos de Hoogendoorn e Bovy [17] e Helbing e Molnár [18], no entanto, conseguiram simular a maioria. São recomendados, portanto, os modelos de força social e os modelos baseados em atividades para a pesquisa de movimento de multidão de pedestres [16].

A terceira área de aplicação corresponde ao layout da infraestrutura dos eventos de multidão, que precisa ser avaliado antes e no curso do evento. O projeto de um evento pode ser auxiliado por um modelo razoavelmente preciso de simulação e razoavelmente rápido. Preciso no sentido de fazer previsões corretas quantos aos locais de alta densidade e pontos de atrito [16]. No entanto, os autores demonstram que os modelos que melhor descrevem o movimento de multidões também apresentam a maior carga computacional. Eles são divididos em modelos cujas tentativas de modelagem microscópica são lentas e precisas e tentativas de modelagem macroscópica rápidas e questionáveis do ponto de vista comportamental. A revisão mostra que os modelos que melhor descrevem o movimento da multidão são também os modelos com maior carga computacional.

\section{O EFEITO ZIGUEZAGUE}

Avaliar a segurança de eventos que reúnem multidões de pedestres tem se mostrado uma tarefa difícil. Além de layouts diferentes de infraestruturas, os movimentos de pedestres também se apresentam diferentes entre os eventos [16]. Os autores afirmam que além da densidade deve-se avaliar a pressão em multidões, capaz de causar risco de morte. A liberação súbita de pressão pode causar estresse e deslocamentos de massa que se assemelham a terremotos de muitos pedestres, podendo gerar situações perigosas [19].

Alguns modelos de multidão de pedestres incorporam interações físicas como as 
pressões criadas pelo contato entre indivíduos em alta densidade. Esses modelos sugerem que características aparentemente sutis da arquitetura podem ter uma grande influência na multidão, particularmente em situações de pânico [6, 7]. Corredores em ziguezague, por exemplo, podem melhorar o fluxo de pedestres ao forçarem a alternância de direção e, consequentemente, da pressão exercida pelos pedestres [7].

Em uma rota de evacuação de emergência, o ideal seria que a multidão não se movesse completamente em linha reta em direção a um gargalo, já que um desenho em ziguezague é capaz de reduzir a pressão na multidão à montante de um gargalo [2]. Também o perigo de cair em escadas pode ser reduzido dividindo-as em pequenos segmentos com direções diferentes, onde o desenho em ziguezague limitaria o acúmulo de pressão em uma multidão e evitaria que pessoas caíssem umas sobre as outras, conforme demonstra a Figura 1 [7].

Figura 1: Design convencional e aprimorado de um estádio.
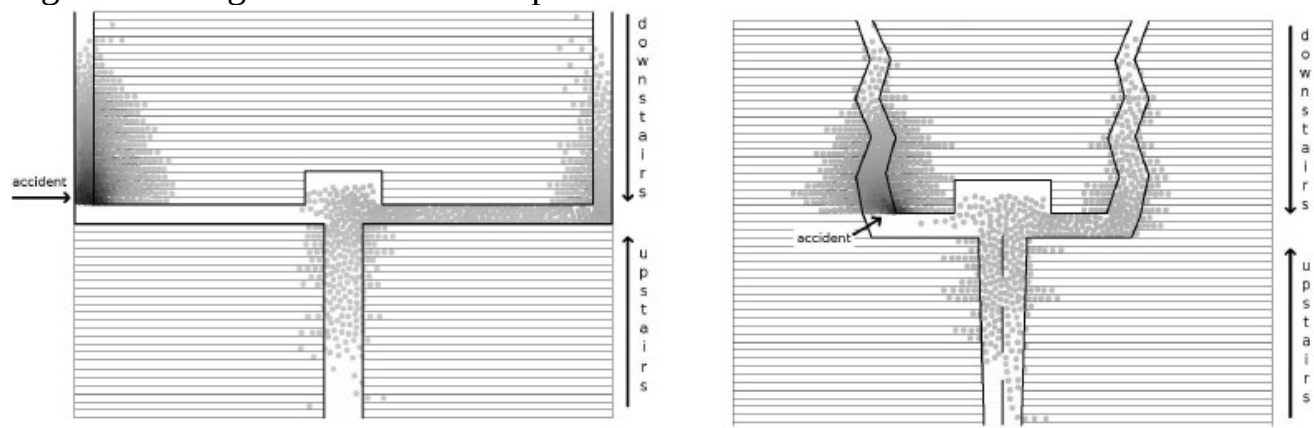

Fonte: Helbing et al. [7].

Vê-se à esquerda, na Figura 1, o projeto convencional de uma saída de estádio em um cenário de emergência. Neste exemplo alguns pedestres caíram no final da escada descendente à esquerda. A cor escura aponta pressões altas, onde os pedestres encontram-se impacientes e empurram uns aos outros por trás. A solução à direita apresenta uma concepção melhorada, com o aumento da largura dos corredores que podem reduzir os tempos de espera e a impaciência (com o mesmo número de assentos), acelerando a evacuação. O ziguezague das escadas muda a direção de empurrão na multidão. Simulações indicam que esse desenho em ziguezague pode reduzir a pressão média na multidão pela metade no local do incidente [7].

Em situações de pânico, estratégias similares também podem ser desenvolvidas [1]. O que é corroborado pelos resultados encontrados em um estudo alemão [3] ao indicarem que percursos em ziguezague são úteis em situações de pânico, ainda que acarretem desconforto, devendo-se analisar o custo/benefício dessa solução para situações normais e de pânico.

\section{MÉTODO DE PESQUISA}

Neste trabalho foram simulados três diferentes projetos de um ambiente fechado, com $300 \mathrm{~m}^{2}$ de área bruta, variando-se o desenho de suas rotas de fuga (Figura 2). As simulações contaram com outras duas importantes variáveis: a velocidade desejada e a densidade de pedestres. Foram simuladas as velocidades desejadas de 1,32 m/s (normal de caminhada) e 2,50 m/s (velocidade de nervosismo) bem como duas densidades de pedestres: 1 e 2 pessoas $/ \mathrm{m}^{2}$. Considerando-se todas as variáveis, contabilizam-se 12 diferentes cenários, simulados 10 vezes cada um deles. 
Conforme a Instrução Normativa [20] consultada para o desenvolvimento deste trabalho, optou-se por simular um ambiente correspondente à Classe de Ocupação "Reunião de público com concentração (locais fechados)”. O cálculo da população foi feito de duas formas:

(i) 1 pessoa $/ \mathrm{m}^{2}$ de área bruta: auditórios ou salas de reunião com mais de $100 \mathrm{~m}^{2}$, teatros, cinemas, óperas, tempos religiosos sem assentos (cadeira, banco ou poltronas);

(ii) 2 pessoas $/ \mathrm{m}^{2}$ de área bruta: boates, clubes noturnos em geral, salões de baile, restaurantes dançantes, bares dançantes, clubes sociais e assemelhados, circos.

Para os casos (i) e (ii) a referida IN indica que a capacidade de corredores e circulação, assim como das portas, deve corresponder a 100 pessoas por unidade de passagem (55 $\mathrm{cm}$ ). Ela indica ainda que acessos, corredores e rotas de fuga devem possuir, no mínimo, 1,65 m de largura para edificações de reunião de público com concentração, além de possuir, no mínimo, duas portas de saída, sendo que uma delas deverá ter a largura mínima de $2 \mathrm{~m}$ e as demais portas complementares conforme a necessidade do dimensionamento. A fim de evitarse variáveis que pudessem interferir na avaliação final das estratégias de projeto analisadas, não foi adotada a largura de $2 \mathrm{~m}$ para uma das saídas, permanecendo ambas com 1,65 m.

Figura 2: Projetos analisados. (a) corredor em ziguezague; (b) corredor reto; (c) painéis em ziguezague antes da saída e corredor reto.

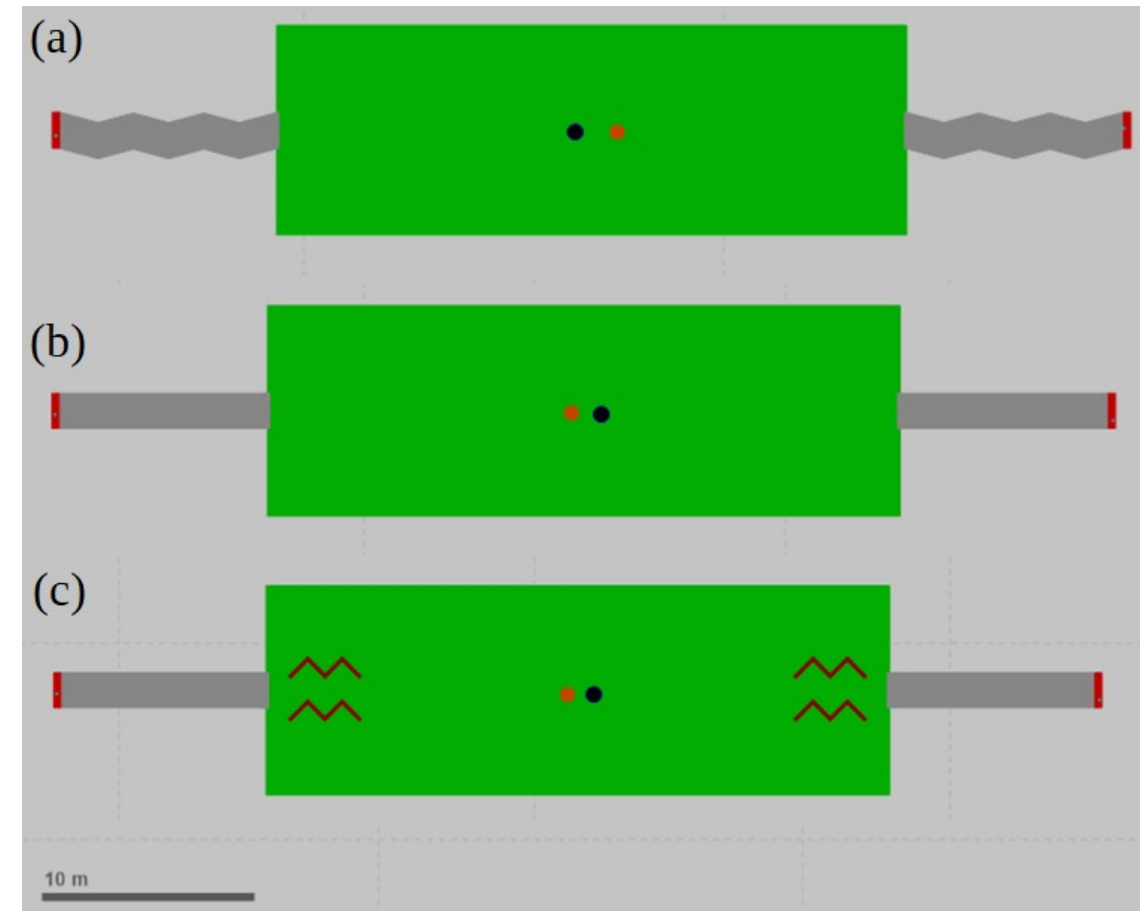

Fonte: Elaborado pelos autores.

O software adotado para as simulações foi o PTV Vissim, em seu módulo PTV Viswalk que se utiliza da abordagem de Força Social. O princípio básico dessa abordagem é a modelagem do ímpeto de movimentação dos pedestres de maneira semelhante à Mecânica Newtoniana, em que o parâmetro de aceleração é resultante de um somatório de forças sociais, psicológicas e físicas relacionadas ao desejo do pedestre de chegar ao seu destino. Inclui ainda a influência de outros pedestres bem como de obstáculos presentes no cenário [21]. A partir da abordagem de Força Social, os pedestres andam independentemente do seu destino, ou seja, não há um modelo de rede pré-definido para as suas trajetórias. 
Em razão da natureza estocástica do modelo de simulação adotado neste trabalho, cujo componente aleatório não pode ser controlado pelo pesquisador [22], ocorrem flutuações capazes de influenciar os resultados em cada simulação realizada. Sendo assim, para fins de análise, considerou-se a média do tempo de evacuação da última pessoa a abandonar o ambiente em cada uma das 10 simulações executadas.

Como parâmetros de simulação utilizou-se o período total de $3.000 \mathrm{~s}$, possibilitando a execução de 10 simulações consecutivas de 300 s cada; a resolução de 10 time step(s) Sim. Sec., fazendo com que o pedestre dê 10 passos por segundo de simulação; semente aleatória 42 que promove a aleatoriedade de pedestres a cada simulação; e velocidade máxima de simulação. O método adotado pelo software padronizada a escolha pelo caminho mais curto para os percursos até o destino final. Os intervalos de tempo utilizados foram: de $0 \mathrm{~s}$ a $300 \mathrm{~s}$, de 300,1 a $600 \mathrm{~s}$, e assim sucessivamente.

Na sequência serão apresentados e discutidos os resultados referentes aos cenários simulados, que foram comparados a partir da determinação de seus fluxos de evacuação (pessoas / segundo).

\section{RESULTADOS E DISCUSSÃO}

A Tabela 1 apresenta os resultados obtidos para cada um dos cenários simulados.

Tabela 1 - Fluxo de evacuação (pessoas/s) em cada cenário analisado

\begin{tabular}{|c|c|c|c|c|}
\hline Cenário & $\begin{array}{l}\text { Projeto analisado } \\
\text { (a), (b), (c) }\end{array}$ & $\begin{array}{c}\text { Velocidade desejada } \\
(\mathrm{m} / \mathrm{s})\end{array}$ & $\begin{array}{l}\text { Densidade } \\
\left(\text { pessoa } / m^{2}\right)\end{array}$ & $\begin{array}{c}\text { Fluxo (pessoas/ } \\
\text { s) }\end{array}$ \\
\hline 1 & (a) & 1,32 & 1 & 2,91 \\
\hline 2 & (b) & 1,32 & 1 & 3,10 \\
\hline 3 & (c) & 1,32 & 1 & 3,00 \\
\hline 4 & (a) & 2,50 & 1 & 9,07 \\
\hline 5 & (b) & 2,50 & 1 & 9,55 \\
\hline 6 & (c) & 2,50 & 1 & 8,47 \\
\hline 7 & (a) & 1,32 & 2 & 2,91 \\
\hline 8 & (b) & 1,32 & 2 & 3,24 \\
\hline 9 & (c) & 1,32 & 2 & 3,28 \\
\hline 10 & (a) & 2,50 & 2 & 10,28 \\
\hline 11 & (b) & 2,50 & 2 & 11,04 \\
\hline 12 & (c) & 2,50 & 2 & 11,18 \\
\hline
\end{tabular}

Fonte: Elaborada pelos autores.

Vê-se, no Gráfico 1, que os resultados mais promissores remetem ao corredor reto. Este Gráfico representa as simulações realizadas com densidade igual a 1 pessoa $/ \mathrm{m}^{2}$. Para a velocidade normal de caminhada $(1,32 \mathrm{~m} / \mathrm{s})$, o projeto (a) mostra-se o menos promissor. Já para a velocidade de nervosismo (2,5 m/s), o projeto (c) apresenta-se como o menos eficaz, com queda mais acentuada do fluxo de pedestres em relação aos demais projetos. 
Gráfico 1 - Fluxo de evacuação para densidade igual a 1 pessoa/m²

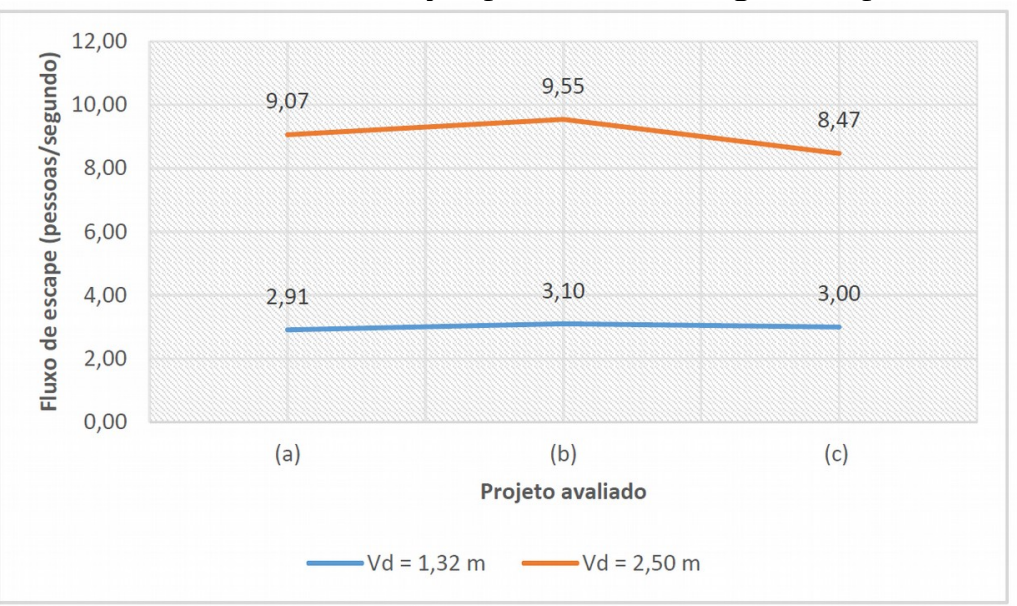

Fonte: Elaborado pelos autores.

O Gráfico 2 apresenta os resultados obtidos a partir das simulações com 2 pessoas $/ \mathrm{m}^{2}$. Para as duas velocidades simuladas vê-se que o projeto (a) foi menos promissor e o projeto (c) o mais eficaz. Essa eficácia ficou mais evidente para a velocidade de $2,5 \mathrm{~m} / \mathrm{s}$ (velocidade de nervosismo).

Gráfico 2: Fluxo de evacuação para densidade igual a 2 pessoas $/ \mathrm{m}^{2}$.

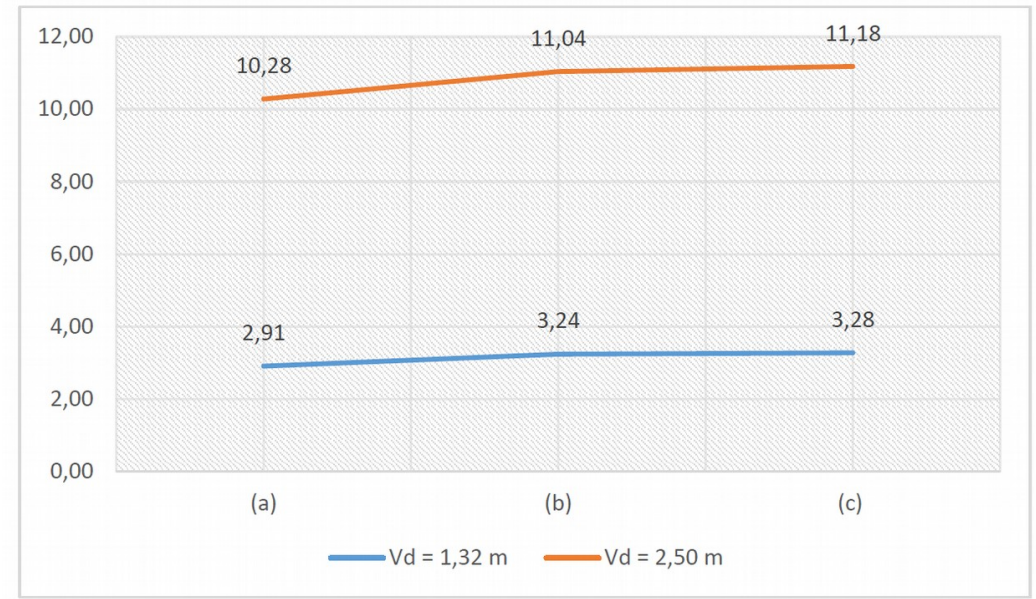

Fonte: Elaborado pelos autores.

A sequência de imagens apresentada nas Figuras 3-6 mostra capturas de tela de simulações dos cenários 10, 11 e 12 em diferentes momentos. As capturas de tela foram realizadas durante a simulação da evacuação de 600 pessoas ( 2 pessoas $/ \mathrm{m}^{2}$ ) na velocidade desejada de 2,5 m/s (velocidade de nervosismo). 
Figura 3: Captura de tela após 1 segundo do início da simulação.

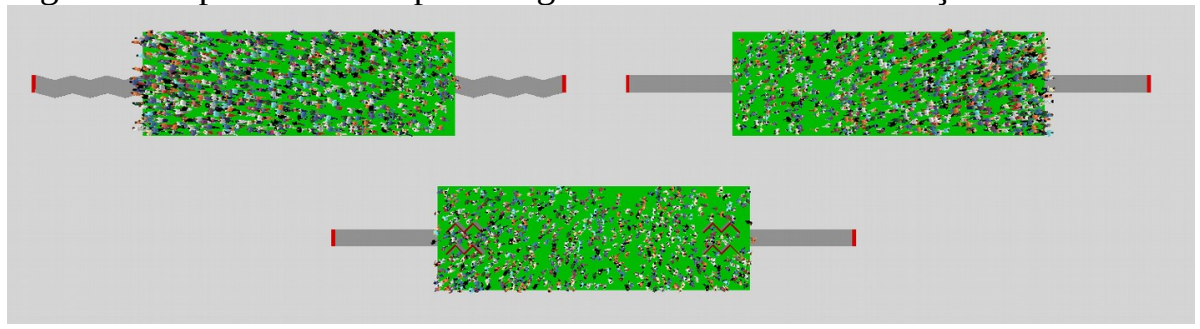

Fonte: Elaborado pelos autores.

Figura 4: Captura de tela após 10 segundos do início da simulação.

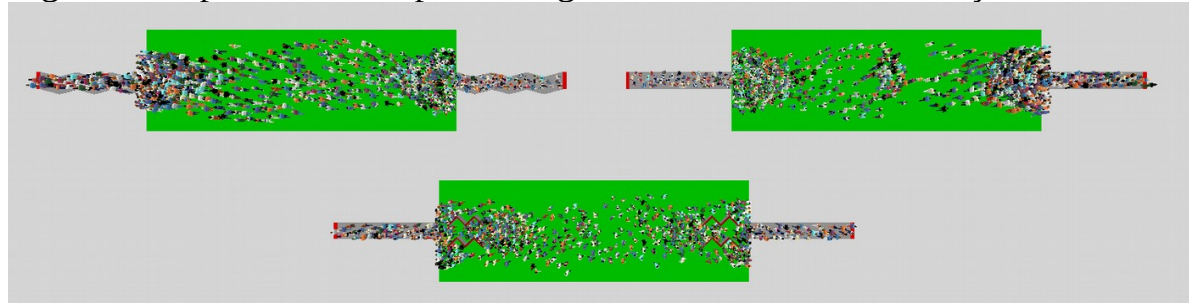

Fonte: Elaborado pelos autores.

Figura 5: Captura de tela após 25 segundos do início da simulação.

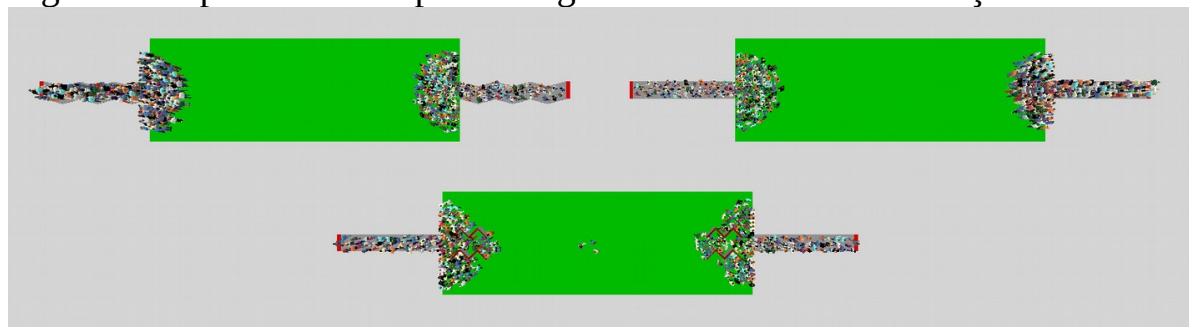

Fonte: Elaborado pelos autores.

Figura 6: Captura de tela após 40 segundos do início da simulação.

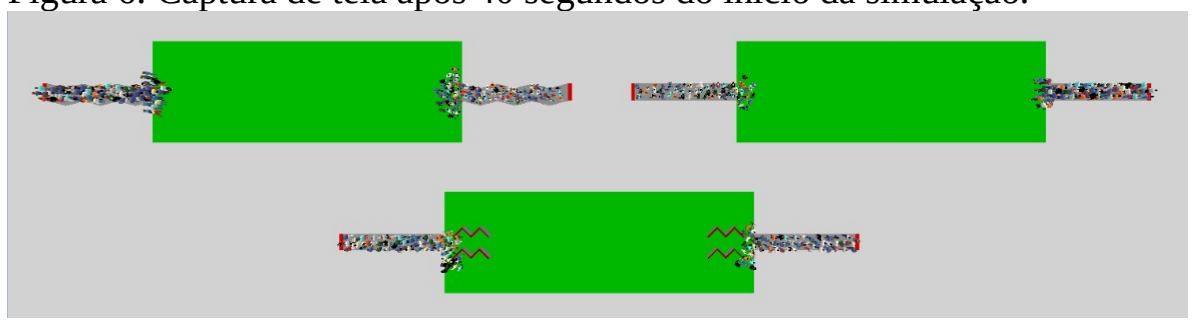

Fonte: Elaborado pelos autores.

É possível observar, especialmente nas Figuras 5 e 6, uma configuração diferenciada no cenário 12 (inferior) quando comparados os arcos formados em frente aos gargalos (representados pelas saídas). O painel em ziguezague posicionado em frente à saída foi capaz de promover um fluxo mais eficaz quando simulados os cenários de maior densidade (2 pessoas $/ \mathrm{m}^{2}$ ).

Os resultados obtidos permitem inferir que o corredor de saída é mais eficaz quando seu desenho se dá em linha reta se comparado ao corredor em ziguezague. No entanto, o painel em ziguezague posicionado em frente às portas de saída atuou de maneira positiva nos cenários cuja densidade era de 2 pessoas $/ \mathrm{m}^{2}$. 


\section{CONSIDERAÇÕES FINAIS}

As simulações realizadas evidenciaram que a presença de percursos em ziguezague após o gargalo (neste estudo representado pelas portas de saída) não foi capaz de gerar fluxos de evacuação mais eficazes. Já a presença do painel em ziguezague antes do gargalo mostrouse eficaz nas situações de maior densidade de pessoas ( 2 pessoas $\left./ \mathrm{m}^{2}\right)$, com redução de até 11,23 \% no tempo total de evacuação. Nesse sentido, pode-se afirmar que a presença de percursos em ziguezague antes dos gargalos foi útil em situações normais e de pânico apenas nos cenários de maior densidade de pessoas. Ou seja, foi ineficaz nos cenários com densidade igual a 1 pessoa $/ \mathrm{m}^{2}$. O que reforça a sua função benéfica, à montante de um gargalo, na alternância da direção e da pressão exercida pelos pedestres em situações de alta densidade. Já a adoção de percursos em ziguezague após os gargalos se mostrou ineficaz em todos os cenários analisados. Destarte, recomenda-se que seja considerado o custo-benefício da adoção dessa estratégia caso a caso, a partir da simulação de possíveis soluções projetuais.

\section{Agradecimentos}

O presente trabalho foi realizado com apoio da Coordenação de Aperfeiçoamento de Pessoal de Nível Superior - Brasil (CAPES) - Código de Financiamento 001 - 88887.091740/2014-01 - Projeto PROALERTAS. Os autores também agradecem ao Grupo PTV pela licença estudantil concedida do software PTV Viswalk.

\section{REFERÊNCIAS BIBLIOGRÁFICAS}

[1] HELBING, D.; FARKAS, I.; MOLNÁR, P.; VICSEK, T. Simulation of Pedestrian Crowds in Normal and Evacuation Situations. In: Schreckenberg, M.; Sharma, S. (eds). Pedestrian and Evacuation Dynamics. Springer, Berlin, p. 21-58, 2002.

[2] HELBING, D.; JOHANSSON, A. Pedestrian, Crowd and Evacuation Dynamics. In: Encyclopedia of complexity and System Science, p. 6476-6495, 2011.

[3] SHUKLA, P. Genetically Optimized Architectural Designs for Control of Pedestrian Crowds. In: KORB, K., RANDALL, M.; HENDTLASS, T. (eds). Artificial Life: Borrowing from Biology. ACAL. Lecture Notes in Computer Science, 5865. Springer, Berlin, Heidelberg, p. 22-31, 2009.

[4] HELBING, D.; MOLNÁR, P. 1995. Social force model for pedestrian dynamics. Physical Review E. v. 51, n. 5, p. 4282-4286, 1995. https://doi.org/10.1103/PhysRevE.51.4282.

[5] HUGHES, R. A continuum theory for the flow of pedestrians. Transportation Research Part B: Methodological, v. 36, n. 6, p. 507-535, 2002. https://doi.org/10.1016/S01912615(01)00015-7.

[6] HELBING, D.; FARKAS I.; VICSEK, T. Simulating dynamical features of escape panic. Nature, v. 407, p. 487-490, 2000.

[7] HELBING, D.; BUZNA, L.; JOHANSSON, A.; WERNER, T. Self-Organized Pedestrian Crowd Dynamics: Experiments, Simulations, and Design Solutions. Transportation Science, v. 39, n. 1, p. 1-24, 2005.

[8] BURD, M.; SHIWAKOTI, N.; SARVI, M.; ROSE. G. Nest architecture and traffic flow: large potential effects from small structural features. Ecological Entomology, v. 35, n. 4, p. 464-468, 2010. https://doi.org/10.1111/j.1365-2311.2010.01202.x. 
[9] GANEM, J. A behavioral demonstration of Fermat's principle. The Physics Teacher, v. 36, n. 2, p. 76-78, 1998. https://doi.org/10.1119/1.880019.

[10] HELBING, D.; KELTSCH, J.; MOLNÁR, P. Modelling the evolution of human trail systems. Nature, v. 388, n. 6637, p. 47-50, 1997.

[11] HENDERSON, L F. The Statistics of Crowd Fluids. Nature, v. 229, n. 5284, p. 381383, 1971.

[12] PREDTETSCHENSKI, W. E; MILINSKI, A. Personenströme in Gebäuden: Berechnungsmethoden für die Projektierung. Rudolf MÄuller, KÄoln-Braunsfeld, 1971.

[13] WEIDMANN, U. Transporttechnik der Fussgäanger. In: Schriftenreihe des Instituts für Verkehrsplanung, Transporttechnik, Straßen- und Eisenbahnbau, ETH Zürich, 1993.

[14] HELBING, D.; FARKAS I.; VICSEK T. Crowd Disasters and Simulation of Panic Situations. In: BUNDE, A.; KROPP, J.; SCHELLNHUBER, H. (eds). Science of Disaster: Climate Disruptions, Heart Attacks and Market Crashes. Springer, Berlin, p. 331-350, 2001.

[15] HENDERSON, L. F. On the Fluid Mechanics of Human Crowd Motion. Transportation Research, v. 8, n.6, p. 509-515, 1974. https://doi.org/10.1016/00411647(74)90027-6.

[16] DUIVES, D.; DAAMEN, W.; HOOGENDOORN, S. State-of-the-art crowd motion simulation models. Transportation research part C: emerging technologies v. 37, p. 193-209, 2013.

[17] HOOGENDOORN, S. P.; BOVY, P. H. L. Pedestrian route-choice and activity scheduling theory and models. Transportation research part B: methodological, v. 38, n. 2, p. 169-190, 2004. https://doi.org/10.1016/S0191-2615(03)00007-9.

[18] HELBING, D.; MOLNÁR, P. Self-organization phenomena in pedestrian crowds. Statistical Mechanics, 1997.

[19] HELBING, D., JOHANSSON, A.; AL-ABIDEEN, H. Z. Dynamics of crowd disasters: an empirical study. Physical review E, v. 75, n. 4, p. $046109,2007$.

[20] SANTA CATARINA. IN 009/DAT/CBMSC - Sistema de Saídas de Emergência. Estabelecer e padronizar critérios de projetos de saída de emergência. Florianópolis: Corpo de Bombeiros Militar De Santa Catarina, 2014. 46 p.

[21] PTV - PLANUNG TRANSPORT VERKEHR AG. PTV VISSIM 9 User's Manual. Karlsruhe: PTV AG, 2016.

[22] LIMA, F.; OLIVEIRA, D.; SAMED, M. Simulação e Cenários como Preparação para Desastres. In: LEIRAS, A.; YOSHIZAKI, H.; SAMED, M.; GONÇALVES, M. (orgs.). Logística Humanitária. 1 ed. Rio de Janeiro: Elsevier, 2017, p. 251-272. 